\title{
Online Calibration for LTE-Based Antenna Array System
}

\author{
Kyunghoon Kim, ${ }^{1}$ Hyunwook Yang, ${ }^{2}$ Jaehyun Jang, ${ }^{3}$ Tiefeng Sun, ${ }^{4}$ \\ Seungwon Choi, ${ }^{1}$ and Jaeho Jung ${ }^{5}$ \\ ${ }^{1}$ School of Electrical and Computer Engineering, Hanyang University, 17 Haengdang-dong, \\ Seongdong-gu, Seoul 133-791, Republic of Korea \\ ${ }^{2}$ System LSI Department, Samsung Electronics Co. Ltd., Banwol-dong, Hwaseong-si, Gyeonggi-do 445-701, Republic of Korea \\ ${ }^{3}$ Commercial Display Research Department Home Entertainment Company, LG Electronics Inc., 222 LG-ro, \\ Jinwi-myeon, Pyeongtaek-si, Gyeonggi-do 451-713, Republic of Korea \\ ${ }^{4}$ Mobile Design Verification Part Mobile SoC Development Department, LG Electronics Inc., 19 Yangjae-daero-11 gil, \\ Seocho-gu, Seoul 137-893, Republic of Korea \\ ${ }^{5}$ Advanced Communications Research Laboratory, ETRI, 218 Gajeong-ro, Yuseong-gu, Daejeon 305-700, Republic of Korea
}

Correspondence should be addressed to Seungwon Choi; choi@dsplab.hanyang.ac.kr

Received 20 April 2016; Accepted 24 October 2016

Academic Editor: Muhammad Ramlee Kamarudin

Copyright (C) 2016 Kyunghoon Kim et al. This is an open access article distributed under the Creative Commons Attribution License, which permits unrestricted use, distribution, and reproduction in any medium, provided the original work is properly cited.

\begin{abstract}
This paper presents a novel calibration method that equalizes the impulse responses of all the Radio Frequency (RF) modules of an antenna array system operating in Long-Term Evolution (LTE) evolved NodeB (eNB). The proposed technique utilizes the Zadoff-Chu (Z-C) sequence of the Primary Synchronization Signal (PSS) and Sounding Reference Signal (SRS) that are available in every LTE data frame for downlink and uplink, respectively, for estimating and compensating the differences in the impulse responses among the RF modules. The proposed calibration method is suitable for wide bandwidth signal environments of LTE because it equalizes the impulse response of each RF module, which is ultimately equivalent to compensate the phase and amplitude differences among RF modules for the entire frequency band. In addition, the proposed method is applicable while the target eNB is transmitting or receiving a data stream. From various experimental tests obtained from a test-bed implemented with 2 RF modules, it has been verified that the proposed method provides a reliable calibration for Release 10 Time Division Duplex (TDD) LTE signals. Phase errors after the calibration in our test-bed have been found to be about $2.418^{\circ}$ and $2.983^{\circ}$ for downlink and uplink, respectively.
\end{abstract}

\section{Introduction}

Massive Multiple Input Multiple Output (MIMO) technology is discussed as a study item of 3rd Generation Partnership Project (3GPP) Release 13 for minimizing the interferences while the communication capacity and transmission distance are maximized [1-3]. Furthermore, the massive MIMO has emerged as a promising solution for realizing the green Information Technology (IT) base-station because the transmitting power can be reduced proportionally to the number of antenna elements in the massive MIMO system [4]. Each RF module of any antenna array system consists of many components such as Analog-to-Digital Converter/Digital-to-Analog Converter (ADC/DAC), filters, amplifiers, and so forth. Since the transfer characteristics of those components at each RF module are in general all different, the transfer characteristics of each RF module in an antenna array system are different from one another. Consequently, in order to fully exploit the merits of a given antenna array system, those transfer characteristics at each RF module should be equalized.

In this paper, we present a novel calibration method that equalizes the impulse responses of all the RF modules in a given antenna array system for beamforming. Since the proposed method unifies the impulse responses of all the RF modules, it is applicable to the wide bandwidth signal environments such as LTE. All the RF modules should exhibit the same transfer characteristics for every subcarrier in a given wide bandwidth. Researches on calibrations for beamforming and Time Division Duplex (TDD) Long-Term Evolution (LTE) implementation have been performed in $[5,6]$ and 


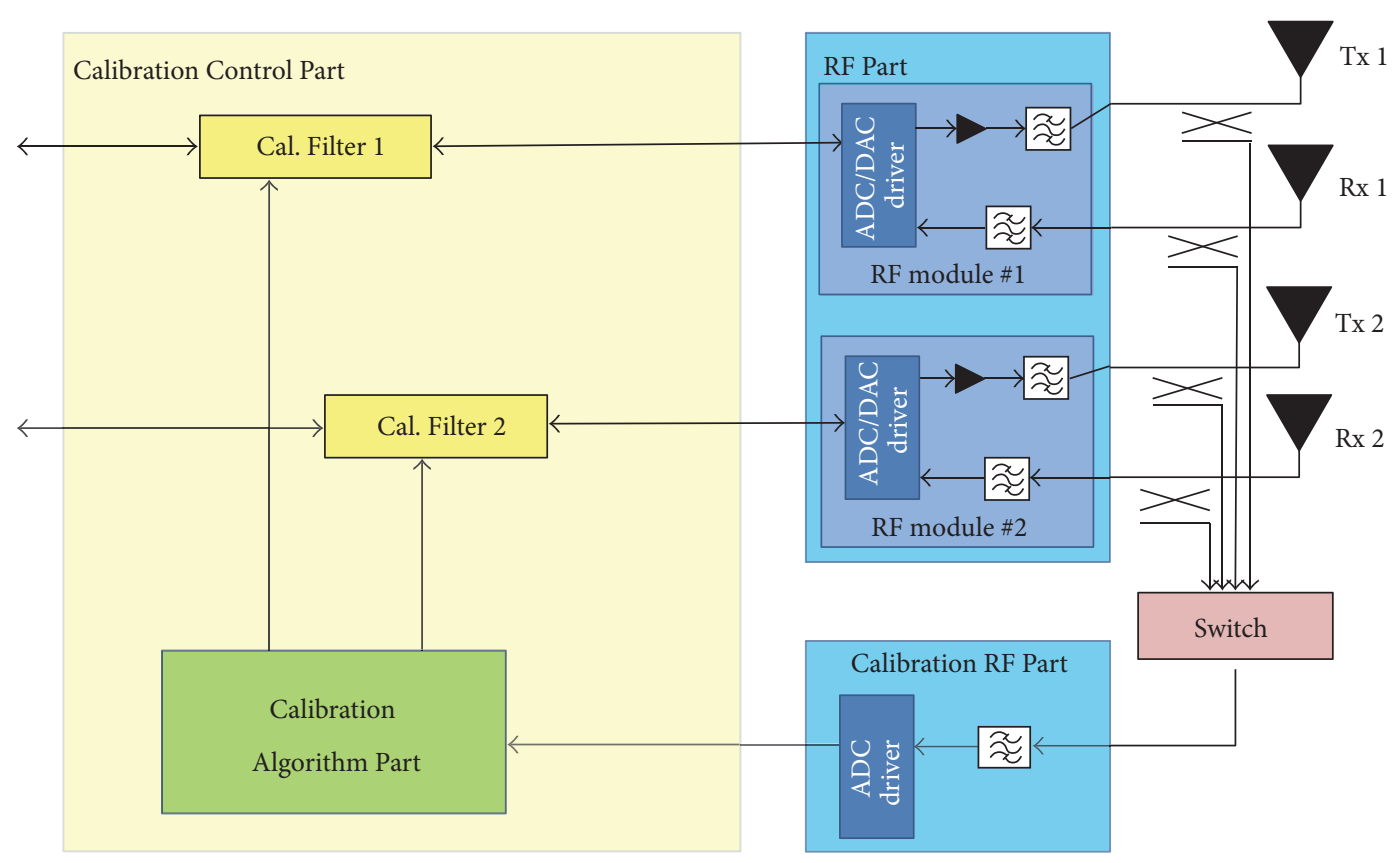

FIGURE 1: Block diagram of proposed calibration system.

$[7,8]$, respectively. However, most conventional calibration methods including the ones shown in [5-8] considered a narrow bandwidth signal environment only, meaning that the impulse response of each RF module consists of a single tap such that the difference in the phase delays among distinct $\mathrm{RF}$ modules can be compensated only for a single subcarrier. In order to apply the methods in [5-8] to wide bandwidth, each of the subcarriers should be considered separately and exhaustively, which would be extremely inefficient. Particularly for mmWave application that is one of the key technologies in 5th Generation (5G) mobile communications, since the bandwidth could be up to a few $\mathrm{GHz}$ [9], it is practically impossible to adopt the calibration techniques given in [5-8]. As a wide bandwidth calibration method, $[10,11]$ introduced procedures of unifying the impulse response of each RF module of a given array system. Since the method shown in $[10,11]$ requires a separate procedure of generating a reference signal with which the impulse response can be estimated, however, it is impossible to apply the method of $[10,11]$ while the array system is under its operating mode. In other words, the array system should be switched off during the procedure of calibrating the RF modules.

The main contribution of this paper is to present a novel calibration method which is applicable to wide bandwidth signal environments such as LTE while the target array system is under the operation of communicating data streams. Performance of the proposed method is verified using a test-bed system implemented with 2 RF modules which transmit and receive the 3 GPP Release 10 TDD LTE signals. The $\mathrm{Z}-\mathrm{C}$ sequence in PSS and SRS is used as a reference signal in the proposed method for downlink and uplink calibration, respectively. Since the Z-C sequence is available in every LTEbased data frame, the proposed technique is applicable without any additional references.
The rest of this paper consists of the following sections. Section 2 introduces a system model considered in this paper. Section 3 explains the proposed calibration method for the downlink and uplink separately. Section 4 introduces the test-bed implemented for verifying the proposed technique. Section 5 concludes this paper.

\section{System Model}

The impulse response of each RF module in a given antenna array system that operates in a wideband signal environment such as LTE can be written as

$$
h_{m}(t)=\sum_{i=0}^{L-1} \alpha_{m, i} \delta\left(t-i \cdot T_{s}\right),
$$

where $\delta(t)$ denotes Dirac delta function, $T_{s}$ and $L$ are the sampling period and number of taps, respectively, and $\alpha_{m, i}$ corresponds to the magnitude of $i$ th tap of $m$ th RF module.

Figure 1 illustrates a block diagram of the calibration system considered in this paper, which consists of RF Part, Calibration RF Part, and Calibration Control Part. In order to verify the feasibility and performance of the calibration method to be shown in the next section, we consider, for simplicity, but without loss of generality, an antenna array system consisting of only 2 RF modules for both transmit and receive array system for LTE evolved NodeB (eNB). The RF Part which consists of the 2 RF modules, as shown in Figure 1, is for both processing the LTE data streams and calibration signals, while the Calibration RF Part and Calibration Control Part are for the calibration only. The RF Part and Calibration RF Part are controlled by the Calibration Control Part for the purpose of calibration. It is noteworthy that, in general, extra hardware is required separately for the calibration of transmit 


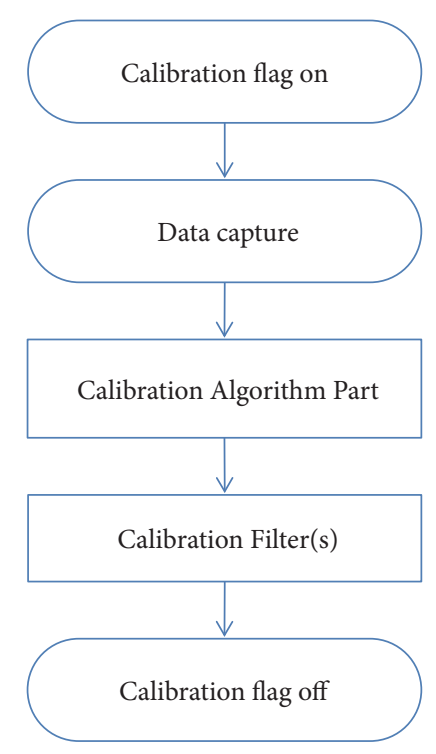

FIgURE 2: Flowchart for the Calibration Control.

and receive RF modules, while the proposed method uses Calibration RF Part shown in Figure 1 for both transmit and receive $\mathrm{RF}$ modules in common. It particularly means that the proposed method is much simpler than conventional methods [12], which require receiving and transmitting modules separately for calibrating transmit and receive RF modules, respectively.

Figure 2 illustrates a flowchart which describes the control of the proposed calibration method. When the calibration flag is on, all the data needed for the calibration are captured and sent to the Calibration Algorithm Part shown in Figure 1. Detailed explanations about the needed data are explained in the next section. The calibration parameters computed in the Calibration Algorithm Part are sent to the Calibration Filter(s). The calibration flag is off when the calibration is completed.

Figure 3 illustrates the amplitude and phase characteristics of the $2 \mathrm{RF}$ modules. The data shown in Figure 3 have been measured in $20 \mathrm{MHz}$ band from the test-bed which has been implemented for verifying the feasibility and performance of the proposed calibration method to be explained in Section 4.

As shown in Figure 3, the 2 RF modules exhibit different characteristics. Note that if the phase differences between the 2 RF modules are exactly the same for all the frequency values, then the calibration does not have to be performed with all the subcarriers exhaustively, meaning that the given signal environments might be considered as a narrow bandwidth. According to Figure 3, the implemented antenna array system seems to exhibit a pretty good coincidence in phase characteristics between the 2 RF modules. More precisely speaking, however, it can be observed in Figure 3 that the values for "A" and "B" are different from each other by about $4^{\circ}$. Taking the inverse Fourier transform of the data points shown in Figure 3, it can further be observed in Figure 4 that the differences in the transfer characteristics between the 2 RF modules result in distinct impulse responses. With the wider observation bandwidth, the differences in the phase characteristics for different frequency points would even be far more conspicuous, which would very keenly emphasize the importance of the wideband calibration. For example, the difference of the phase characteristics for the bandwidth of $500 \mathrm{MHz}$ has been found to be up to $90^{\circ}[10,11]$.

Figure 4 illustrates the impulse responses of the $2 \mathrm{RF}$ modules, which have been obtained by taking the inverse Fourier transform of the data point shown in Figure 3. As shown in Figure 4, the impulse responses involve multitaps. Note that most conventional calibration methods [5-8] assumed that the impulse responses of all the RF modules consist of a single tap. As shown in Figure 4, however, the actual impulse responses of RF modules involve multitap; thus, the given signal environment should be considered as being wideband.

\section{Proposed Calibration Algorithm}

In this section, a novel procedure is proposed for calibrating each RF path of antenna array system at eNB of LTE. The new technique equalizes the impulse response of each of the $\mathrm{RF}$ paths in a given antenna array system while the eNB is under the operation of receiving or transmitting LTE signals. Reference signal used for the proposed calibration procedure is the Z-C sequence of PSS and SRS for downlink and uplink, respectively, which is included in every LTE data frame. Normally, in LTE systems, the Z-C sequence in PSS is used for acquiring the Subframe Synchronization during downlink, while that in SRS is for capturing the Channel Quality Indicator (CQI) during uplink. The calibration method proposed in this paper utilizes the $\mathrm{Z}$ - $\mathrm{C}$ sequence in order to first estimate and then equalize the impulse response of each of the RF paths of the antenna array system in a given eNB.

3.1. Downlink Calibration (Tx Calibration). Figure 5 illustrates a block diagram describing the proposed method for downlink, meaning that $\mathrm{eNB}$ is in transmitter (Tx) mode. The objective is to design the Calibration Filter shown in Figure 5 in such a way that the cascade of the Calibration Filter and the 2nd Transmitter of which the impulse response is $h_{t, 2}(n)$ becomes equivalent to the impulse response of the 1st Transmitter, that is, $h_{t, 1}(n)$.

The 1st Transmitter and 2nd Transmitter shown in Figure 5 correspond to the RF modules \#1 and \#2, respectively, shown in Figure 1. In addition, during the downlink calibration, the Calibration RF Part shown in Figure 1 operates as a receiver. For the downlink calibration, the Calibration Algorithm Part needs 4 inputs signals described as follows.

Input 1. LTE data stream to be transmitted through the 1st antenna element, $x_{1}(n)$, becomes the 1st input signal, (1), of Calibration Algorithm Part shown in Figure 5.

Input 2. The $x_{1}(n)$ flows through the 1st Transmitter of RF Part and the Receiver of Calibration RF Part to become the 2nd input of Calibration Algorithm Part, that is, $h_{\text {ref }}(n) * h_{t, 1}(n) *$ $x_{1}(n)$, which is denoted as (3)-1 in Figure 5. 

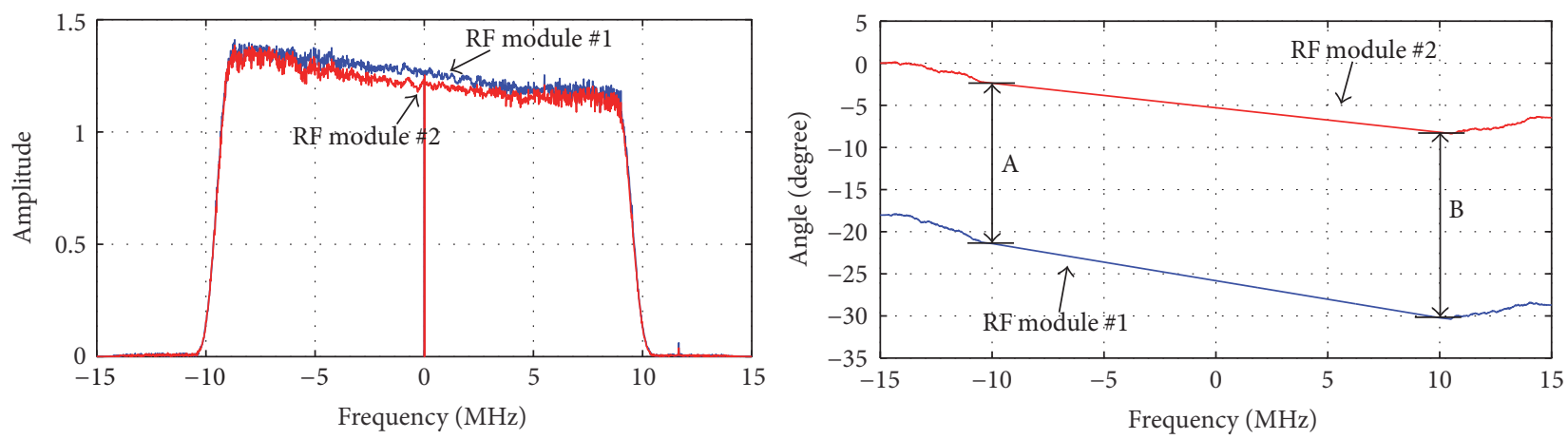

FIgURE 3: Amplitude/phase of the 2 RF modules measured in the implemented test-bed.

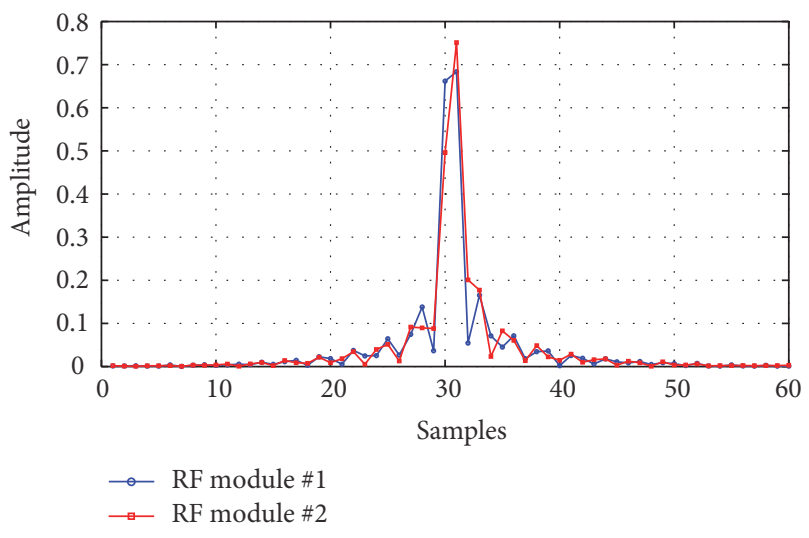

FIGURE 4: Impulse responses of the 2 RF modules.

Input 3. LTE data stream to be transmitted through the $2 \mathrm{nd}$ antenna element, $x_{2}(n)$, becomes the 3rd input signal, (2), of Calibration Algorithm Part shown in Figure 5.

Input 4 . The $x_{2}(n)$ flows through the 2 nd Transmitter of RF Part and the Receiver of Calibration RF Part to become the 4 th input of Calibration Algorithm Part, that is, $h_{\text {ref }}(n) *$ $h_{t, 2}(n) * x_{2}(n)$, which is denoted as (3)-2 in Figure 5. Note that the Calibration Filter in this case is bypassed.

Switch 1 and Switch 2 in Figure 5 have to be set properly in such a way that the inputs of Calibration Algorithm Part are determined in the order of Input 1 to Input 4 as explained above.

The operation of Frame Synchronization of Calibration Algorithm Part shown in Figure 5 is based on the PSS of each LTE data frame. The PSS $d_{u}(n)$ consists of a Z-C sequence of length 62 as follows:

$$
d_{u}(n)= \begin{cases}e^{-j(\pi u n(n+1) / 63)} & \text { for } n=0,1, \ldots, 30 \\ e^{-j(\pi u(n+1)(n+2) / 63)} & \text { for } n=31,32, \ldots, 61,\end{cases}
$$

where the subscript $u$ denotes the $\mathrm{Z}$ - $\mathrm{C}$ root sequence index, which is one of 25,29 , or 34 [13].

Figure 6 illustrates LTE data frame architecture which shows how the PSS is mapped in each data frame of TDD LTE system [12]. As shown in Figure 6, each data frame is $10 \mathrm{~ms}$ long and consists of 10 subframe while each subframe consists of 2 slots with each slot being composed of 7 OFDM symbols. The PSS is assigned at the 3rd symbol of the 1st slot of the 2nd subframe and 3rd OFDM symbol of the 1st slot of the 7th subframe, which is $5 \mathrm{~ms}$ apart from each other. In spectral domain, PSS includes 63 subcarriers at the center of the corresponding OFDM symbol.

Figure 7 illustrates a detailed block diagram of Frame Synchronization shown in Figure 5. Through Switch 2, data samples corresponding to the length of 1 frame, that is, $10 \mathrm{~ms}$, are stored in the order of (1), (3)-1, (2), and (3)-2 as explained earlier. The samples stored in the Buffer, of which the Z-C sequence's index must be one of 25,29 , or 34 , and the Z-C sequence shown in (2) are correlated with each other. When the $\mathrm{Z}-\mathrm{C}$ index in the $\mathrm{Z}$-C Buffer is coincident with that of the Z$\mathrm{C}$ sequence of the data samples in the Buffer, the maximum correlation can be obtained; thus, the Start Point can be obtained.

In the box denoted as Remove CP in Figure 5, 144 samples corresponding to the $\mathrm{CP}$ are removed from the Start Point, leaving the LTE data part only. If the data $x(n)$ is a combination of Tx data $s(n)$ and CP, then we can obtain (1) $s_{1}(n)$, (2) $s_{2}(n)$, (3) $-1 s_{1}(n) * h_{t, 1}(n) * h_{\text {ref }}(n)$, and (3) $-2 s_{2}(n) * h_{t, 2}(n) *$ $h_{\text {ref }}(n)$ after the Remove CP. These signals, (1), (2), (3)-1, and (3)2, are then 2,048-point FFT in the FFT block and transferred to the Buffer \& Divider block shown in Figure 5. From the Divider, we obtain the following:

$$
\frac{S_{1}[k] H_{t, 1}[k] H_{\text {ref }}[k] / S_{1}[k]}{S_{2}[k] H_{t, 2}[k] H_{\text {ref }}[k] / S_{2}[k]}=\frac{H_{t, 1}[k]}{H_{t, 2}[k]},
$$

where upper case letters correspond to the Fourier transform of lower case letters.

Taking the inverse Fourier transform of the right-hand side of (3), we obtain $h_{t, 1}(n) * h_{t, 2}^{-1}(n)$ which is used as the impulse response of the Calibration Filter shown in Figure 5, which means $x_{2}(n)$ becomes $h_{t, 1}(n) * h_{t, 2}^{-1}(n) * x_{2}(n)$ after the Calibration Filter. Consequently, the cascade of the Calibration Filter and 2nd Transmitter becomes the same as the impulse response of the 1st Transmitter, $h_{t, 1}(n)$; thus the impulse responses of both RF modules become equivalent.

3.2. Uplink Calibration (Rx Calibration). Figure 8 illustrates a block diagram describing the proposed method for uplink, 


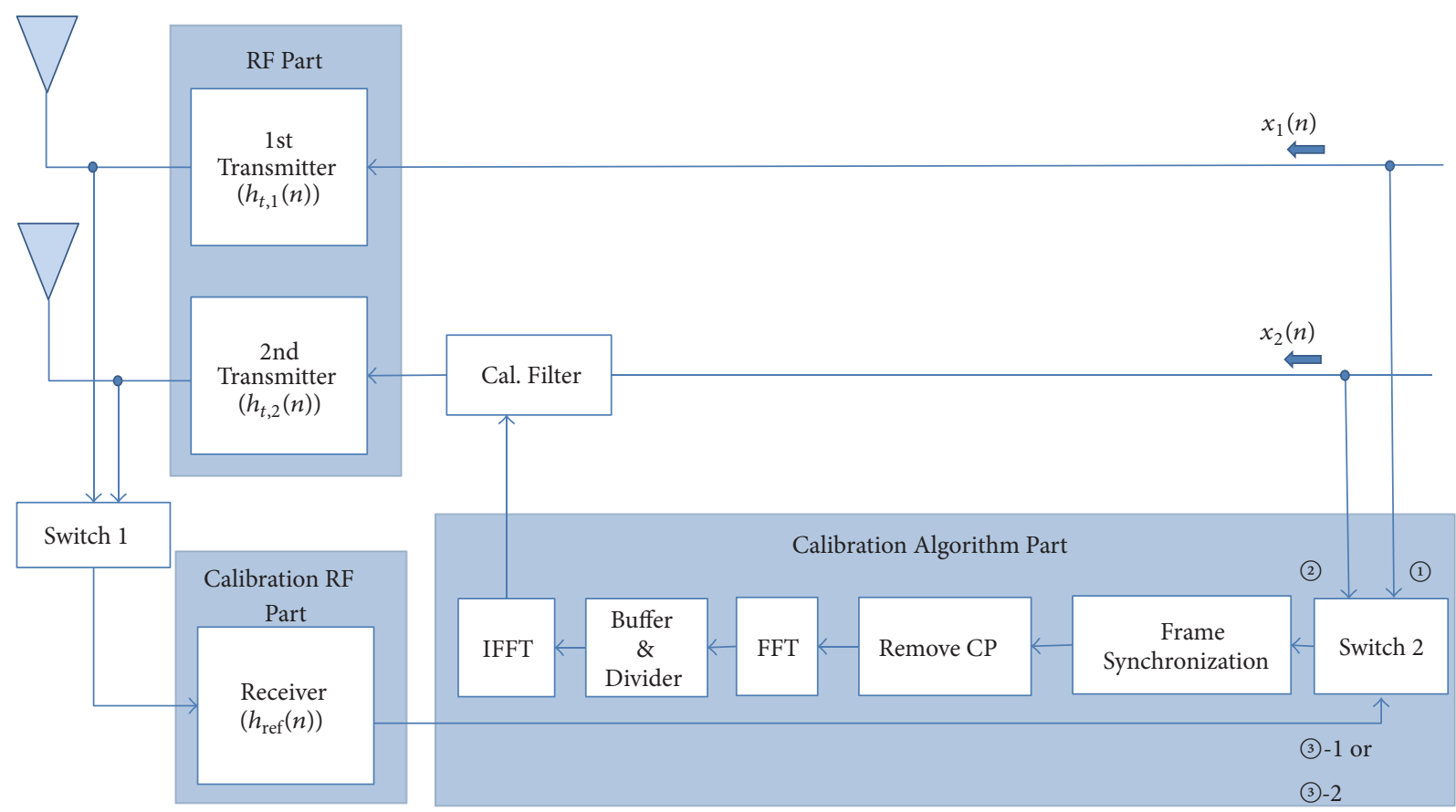

FIGURE 5: Diagram of the proposed downlink calibration.

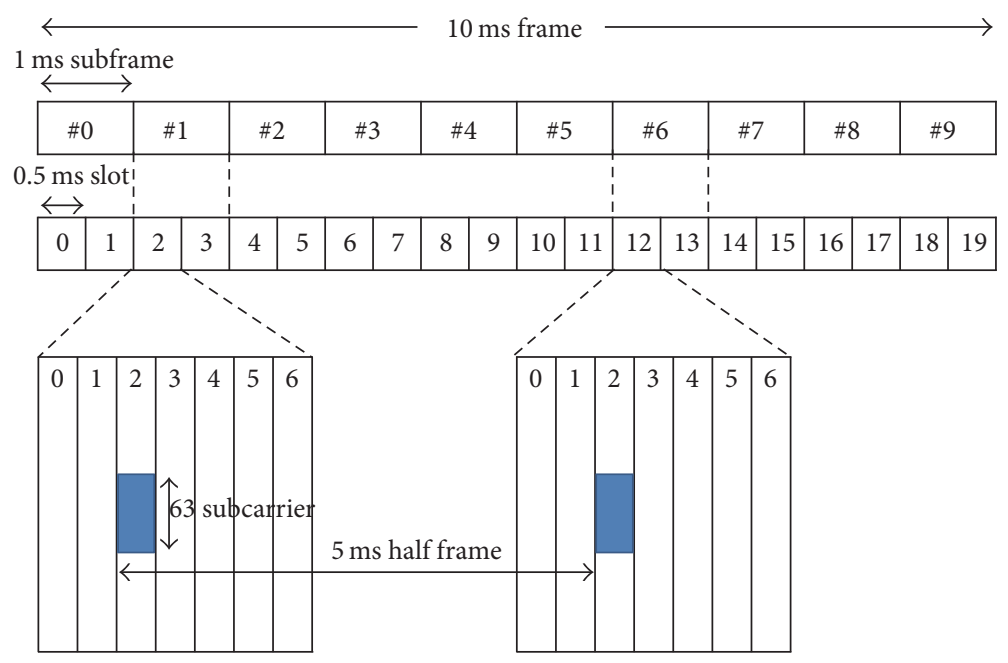

$d_{u}(n)$, PSS (Primary Synchronization Signal)

FIGURE 6: Architecture of downlink LTE frame.

meaning that eNB is in receiver $(\mathrm{Rx})$ mode. The objective is to design the Calibration Filters 1 and 2 shown in Figure 8 in such a way that the cascade of the 1st Receiver of which the impulse response is $h_{r, 1}(n)$ and Calibration Filter 1 and that of the 2 nd Receiver of which the impulse response is $h_{r, 2}(n)$ and Calibration Filter 2 becomes equivalent to each other.

The 1st and 2nd Receiver shown in Figure 8 correspond to the RF modules \#1 and \#2, respectively, shown in Figure 1. In addition, during the uplink calibration as well as in the case of downlink calibration, the Calibration RF Part shown in Figure 1 operates as a receiver. For the uplink calibration, the
Calibration Algorithm Part needs 4 inputs signals described as follows.

Input 1. LTE data stream received through the 1st antenna element, $r_{1}(t)$, after passing through the Receiver of Calibration RF Part by way of Switch 1 becomes the 1st input signal, (1-1, that is, $h_{\text {ref }}(n) * r_{1}(n)$, of Calibration Algorithm Part shown in Figure 8.

Input 2. The $r_{1}(n)$ flows through the 1st Receiver of RF Part to become the 2nd input of Calibration Algorithm Part, that is, $h_{r, 1}(n) * r_{1}(n)$, which is denoted as (2) in Figure 8. 


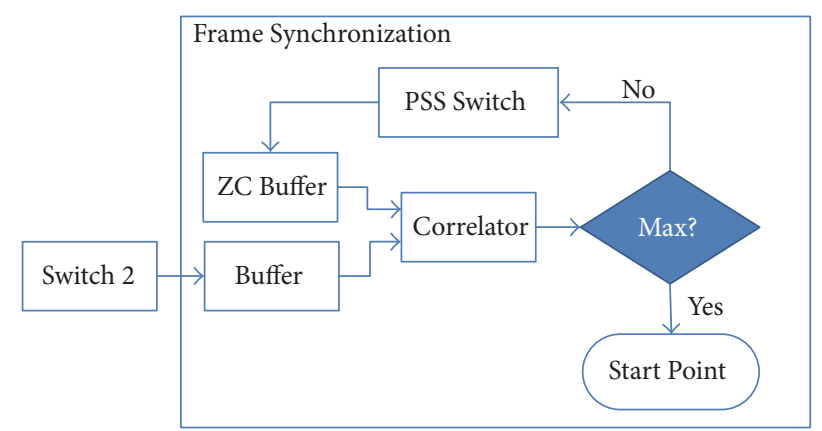

FIgURE 7: Detailed diagram of Frame Synchronization.

Input 3. LTE data stream received through the 2nd antenna element, $r_{2}(t)$, after passing through the Receiver of Calibration RF Part by way of Switch 1 becomes the 3rd input signal, (1)-2, that is, $h_{\text {ref }}(n) * r_{2}(n)$, of Calibration Algorithm Part shown in Figure 8.

Input 4. The $r_{2}(n)$ flows through the 2 nd Receiver of RF Part to become the 4 th input of Calibration Algorithm Part, that is, $h_{r, 2}(n) * r_{2}(n)$, which is denoted as (3) in Figure 8 .

Switch 1 and Switch 2 in Figure 8 have to be set properly in such a way that the inputs of Calibration Algorithm Part are determined in the order of Input 1 to Input 4 as explained above. Then, the signals (1)-1, (2), (1)-2, and (3) are successively stored in the Receive Buffer under the control of Switch 1 and Switch 2.

As mentioned earlier, the uplink calibration introduced in this paper is based on SRS, while the downlink calibration utilizes PSS. Although the transmit interval and Z-C sequence of the SRS are user-dependent, eNB knows both the transmit interval and $\mathrm{Z}-\mathrm{C}$ sequence of the SRS for each user. In this paper, for simplicity but without loss of generality, we assume only 1 user in a given cell whose SRS sequence is stored in the SRS Buffer of the given eNB. Computing the correlation between the SRS Buffer and Receive Buffer and taking Fourier transform on the results of the correlations, we can obtain $H_{\text {ref }}[k] R_{1}[k], H_{r, 1}[k] R_{1}[k], H_{\text {ref }}[k] R_{2}[k]$, and $H_{r, 2}[k] R_{2}[k]$ as a result of correlation with (1)-1, (2), (1)-2, and (3), respectively. Using those 4 values, we obtain the following values from the Divider and IFFT shown in Figure 8:

$$
\begin{aligned}
& \frac{R_{1}[k] H_{\text {ref }}[k]}{R_{1}[k] H_{r, 1}[k]} \stackrel{\text { IFFT }}{\longrightarrow} h_{\text {ref }}(n) * h_{r, 1}^{-1}(n), \\
& \frac{R_{2}[k] H_{\text {ref }}[k]}{R_{2}[k] H_{r, 2}[k]} \stackrel{\text { IFFT }}{\longrightarrow} h_{\text {ref }}(n) * h_{r, 2}^{-1}(n) .
\end{aligned}
$$

Consequently, using the right-hand side terms of (4) as the impulse responses of the Calibration Filers 1 and 2, respectively, the impulse responses of RF modules in uplink can all be equalized to the impulse response of the Calibration RF Filter, $h_{\text {ref }}(n)$.

Since the proposed calibration method introduced in this section unifies all the impulse responses of RF modules, it can be used not only for a narrow band signal environment where
TABLE 1: System parameters.

\begin{tabular}{lc}
\hline System parameter & Value \\
\hline Communication standard & 3GPP Rel. 10 TDD LTE \\
System bandwidth & $20 \mathrm{MHz}$ \\
FFT/iFFT size & 2,048 \\
Number of RF modules $(m)$ & 2 \\
Zadoff-Chu root sequence index $(u)$ & 25 \\
Estimated number of each impulse & 75 \\
response's taps $(L)$ & $32.52 \mathrm{nsec}$ \\
Sampling period $\left(T_{s}\right)$ &
\end{tabular}

the calibration for a single subcarrier can compensate the phase differences among the RF modules but also for a wideband signal environment such as LTE where the phase compensation is required for each of all the subcarriers exhaustively. Instead of using each of all the subcarriers as a reference signal for calibrating the RF modules, the proposed method unifies the impulse responses of all the RF modules using the $\mathrm{Z}$-C sequence as a reference signal, where the $\mathrm{Z}-\mathrm{C}$ sequence is available at every LTE data frame. Furthermore, the proposed calibration is applicable while the antenna array system of a given eNB is under operation of transmitting or receiving the LTE data sequences, which we call online calibration.

\section{Experimental Results}

In this section, we present a test-bed antenna array system consisting of 2 RF modules which is a part of TDD LTE eNB system. The test-bed system has been implemented for verifying the feasibility of the proposed calibration method. The test-bed system operates as Figures 5 and 8 during downlink and uplink, respectively. Table 1 summarizes the system parameters of the test-bed system.

Communication standard of the data frame used in our test-bed is 3GPP Release 10 TDD LTE [12] which is fed from MATLAB signal into our test-bed system properly for downlink and uplink operations as shown in Figures 5 and 8, respectively, except that the antenna elements are excluded in the test-bed, meaning that the signals are fed through Ethernet instead of antennas. The number of taps, $L$, and sampling period, $T_{s}$, introduced in (1) have been set up to 75 and $32.52 \mathrm{nsec}$, respectively, and the $\mathrm{Z}-\mathrm{C}$ root sequence index root $u$ [12] shown in (2) has been set up to 25 .

Figure 9 illustrates the photograph of our test-bed implemented for verifying the feasibility and performance of the proposed calibration method. As mentioned earlier in Figure 1, our test-bed consists of 3 parts, RF Part, Calibration RF Part, and Calibration Control Part. The Calibration Control Part, as mentioned in Sections 2 and 3, controls Switch 1 and Switch 2, Calibration Filter, and so forth. Both Calibration RF Part and RF Part consist of 4 subparts as follows. First subpart consists of antenna elements, RF bandpass filter, circulator, and couplers for capturing RF signals into the RF Calibration Part. Second subpart includes power amplifiers and analog predistorter. Third subpart includes a clock synthesizer and low-noise-amplifier. Fourth subpart includes interfaces for 


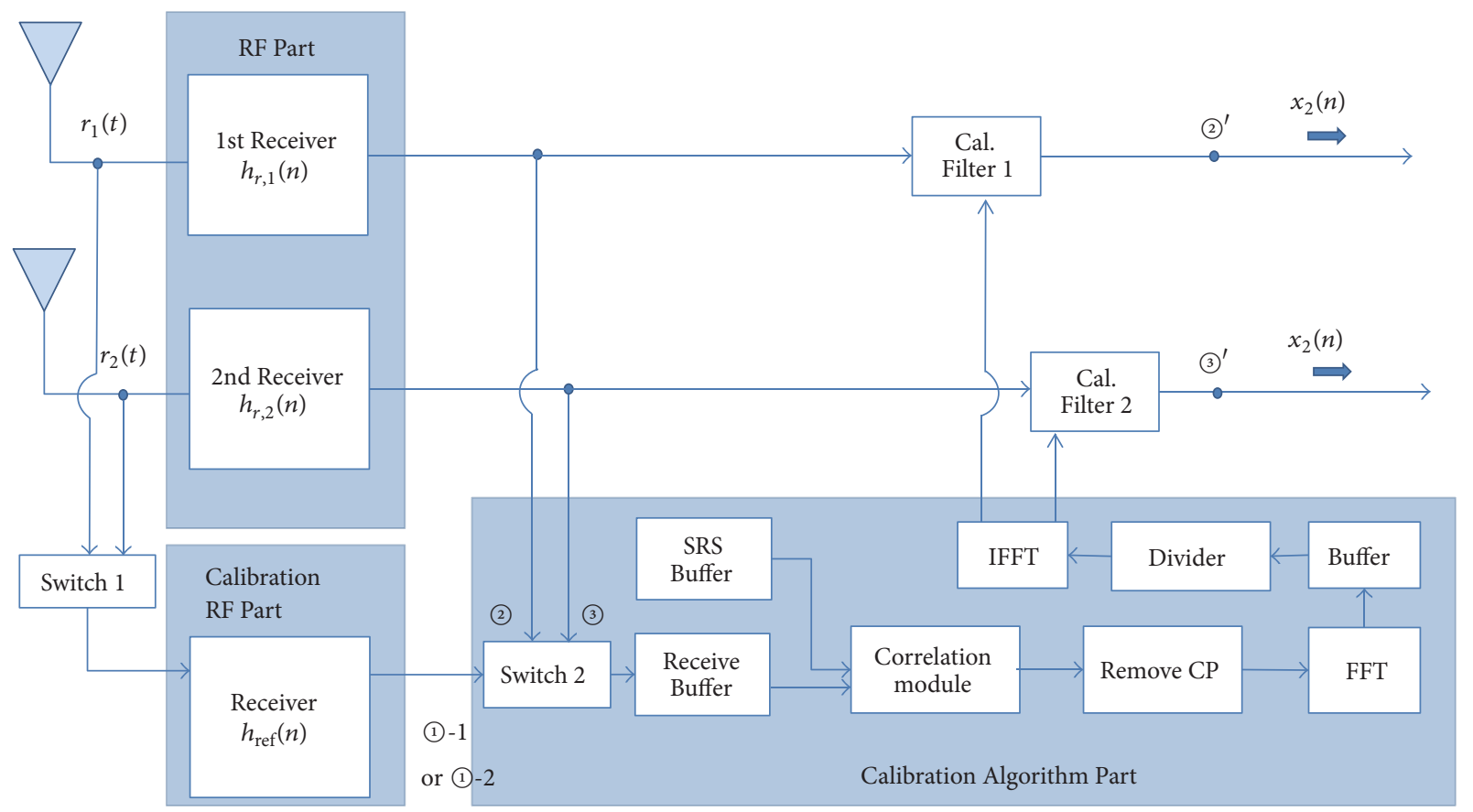

FIGURE 8: Diagram of the proposed uplink calibration.

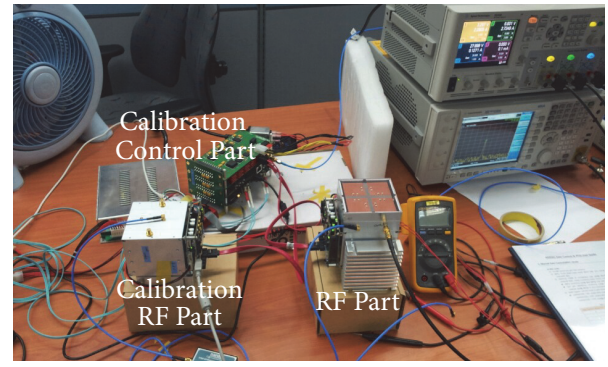

FIgURE 9: Photograph of implemented test-bed.

the RF Calibration Part or RF Part to communicate with the Calibration Control Part.

Figure 10 illustrates the impulse responses of the $2 \mathrm{RF}$ modules in the test-bed system measured before and after the proposed calibration method for both downlink and uplink. Since the impulse function itself cannot be used for measuring the impulse response, we use the correlation between the prestored $\mathrm{Z}-\mathrm{C}$ sequence and $\mathrm{Z}-\mathrm{C}$ sequence which passes through each of the 2 RF modules. As shown in Figure 10, the impulse responses of the $2 \mathrm{RF}$ modules are quite different from each other before the calibration is applied for both downlink and uplink, while they become quite comparable to each other after applying the proposed calibration method.

Figure 11 illustrates the in-phase (I) and quadrature (Q) signal components captured at the $2 \mathrm{RF}$ modules before applying the proposed calibration method. To obtain the data points shown in Figure 11, we have first turned off the calibration flag and captured (3)-1 and (3)-2 after applying the same LTE data stream for both $x_{1}(n)$ and $x_{2}(n)$ shown in Figure 5. It can be observed that, due to the mismatch between the 2
RF modules, both I and Q components of the 2 RF modules are quite different from each other.

Figure 12 illustrates the I and Q signal components captured at the 2 RF modules after applying the proposed calibration method. The procedure of obtaining the signal components shown in Figure 12 is exactly the same as that of Figure 11 except that the calibration flag is turned on at the beginning of the procedure for obtaining the data points shown in Figure 12. As shown in Figure 12, the data points measured at both RF modules are very well coincident with each other due to the calibration. While Figures 11 and 12 show the situations in the downlink, we have observed the similar situations in uplink.

The performance of the proposed calibration method has been analyzed by computing the phase error between the 2 RF modules, which can be obtained as [14]

$$
\begin{aligned}
& \text { phase error }=E\left[\arctan \left(\frac{\left|\operatorname{image}\left(c_{1}(n)\right)\right|}{\left|\operatorname{real}\left(c_{1}(n)\right)\right|}\right)\right. \\
& \left.-\arctan \left(\frac{\left|\operatorname{image}\left(c_{2}(n)\right)\right|}{\left|\operatorname{real}\left(c_{2}(n)\right)\right|}\right)\right],
\end{aligned}
$$

where $E[\cdot]$ denotes an expectation of $\cdot$ and $c_{1}(n)$ and $c_{2}(n)$ are (3)-1 and (3)-2 which are shown in Figure 5, respectively, in the downlink or (2) and (3) which are shown in Figure 8, respectively, in the uplink. To obtain the phase error between the 2 RF modules accurately, we have considered about 900,000 data samples of I and Q. Before the calibration process, the phase errors in down and uplink of the test-bed were about $18.676^{\circ}$ and $19.988^{\circ}$, respectively. The phase errors computed after calibration in our test-bed have been found to be about $2.418^{\circ}$ and $2.983^{\circ}$ for downlink and uplink, respectively. 

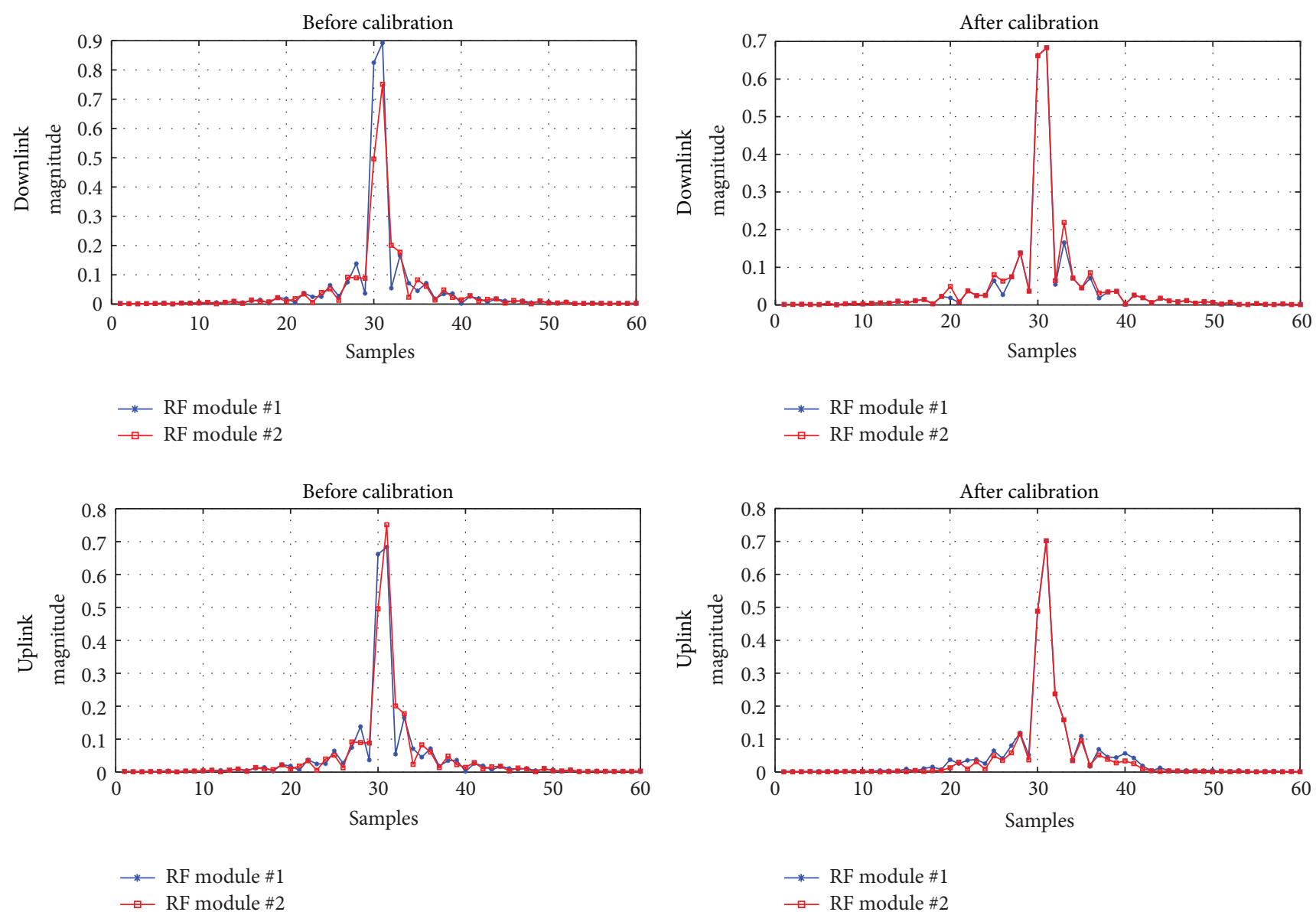

$\rightarrow$ RF module \#1

$\rightarrow$ RF module \#2

FIGURE 10: Impulse responses of the 2 RF modules before and after the proposed calibration.

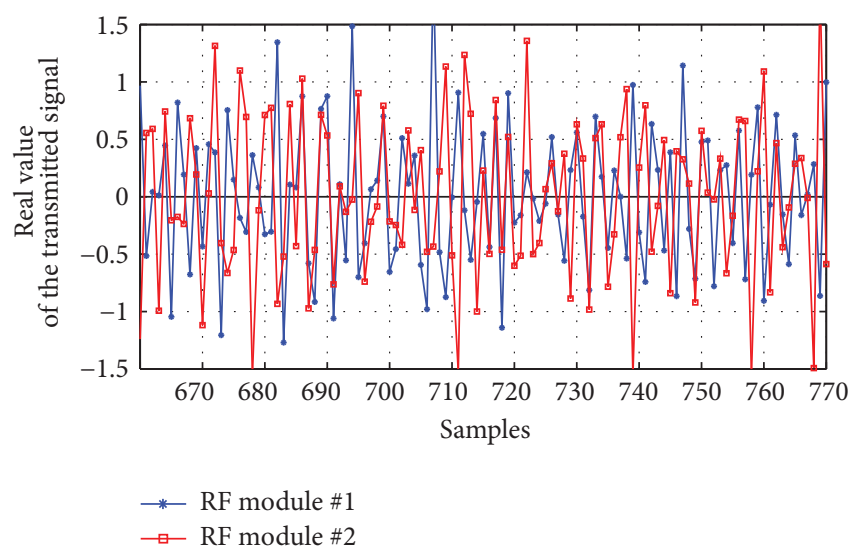

(a) In-phase signal components

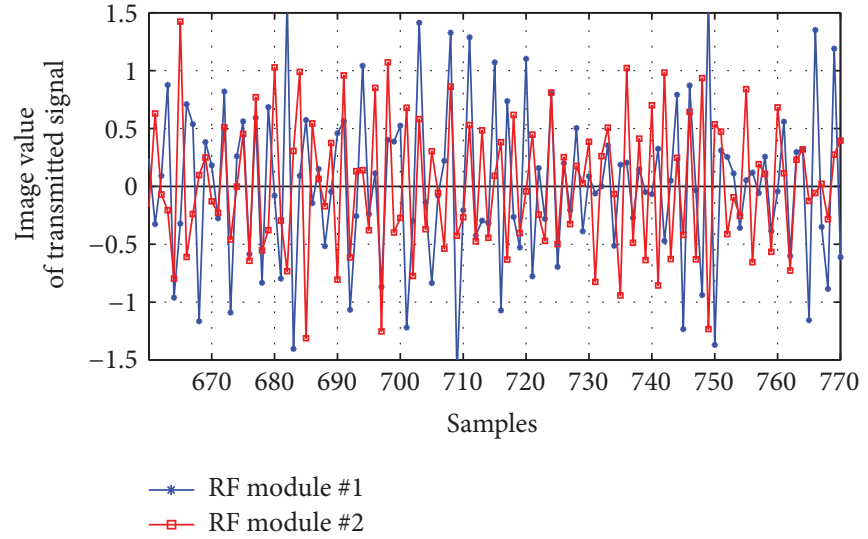

(b) Quadrature signal components

FIGURE 11: In-phase and quadrature signal components captured before calibration during downlink.

Table 2 shows the phase error as a function of SNR. Note that since the SNR in each RF module cannot easily be controlled, the values shown in Table 2 have been obtained from computer simulations instead of experimental tests, which implies that the SNR in our test-bed is a little less than $30 \mathrm{~dB}$. Phase error associated with the proposed method has been compared to that of [11], which can also be used in a wide bandwidth signal environment, as shown in Table 2. Both methods result in comparable performances. However, as mentioned earlier in Chapter 1, the method of [11] cannot be applied in the operating mode, which means the array system should be switched off during the procedure of calibrating the RF modules for the method of [11], whereas the proposed method can be applied while the array system is in its operation mode. 


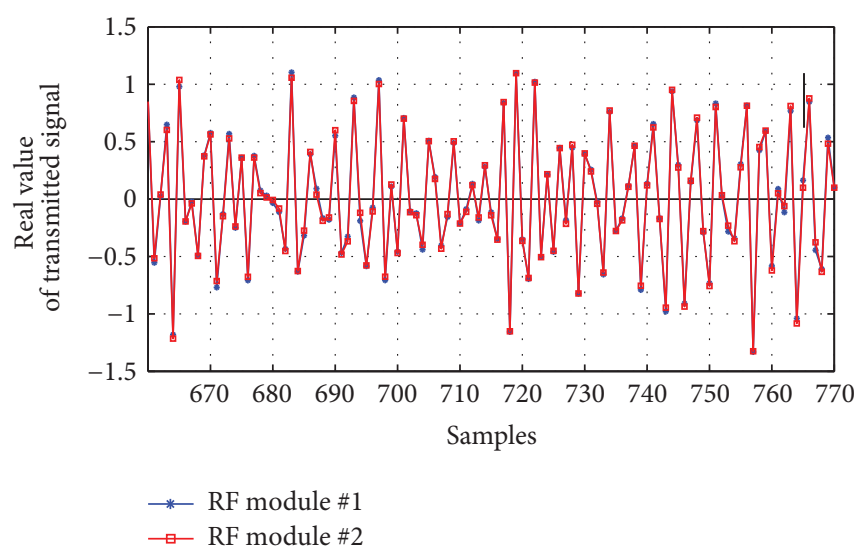

(a) In-phase signal components

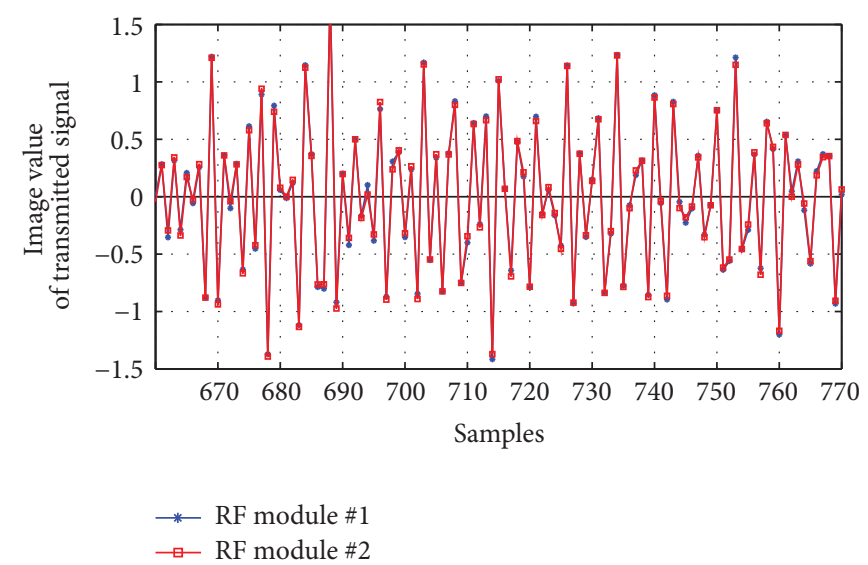

(b) Quadrature signal components

FIGURE 12: In-phase and quadrature signal components captured after calibration during downlink.

TABLE 2: Phase error according to SNR.

\begin{tabular}{|c|c|c|c|c|c|c|}
\hline \multirow{2}{*}{ Item } & & \multicolumn{5}{|c|}{ SNR } \\
\hline & & $10 \mathrm{~dB}$ & $15 \mathrm{~dB}$ & $20 \mathrm{~dB}$ & $25 \mathrm{~dB}$ & $30 \mathrm{~dB}$ \\
\hline \multirow{2}{*}{ Proposed calibration method } & Downlink & $3.729^{\circ}$ & $3.350^{\circ}$ & $3.207^{\circ}$ & $3.083^{\circ}$ & $2.202^{\circ}$ \\
\hline & Uplink & $4.395^{\circ}$ & $4.031^{\circ}$ & $3.366^{\circ}$ & $3.067^{\circ}$ & $2.810^{\circ}$ \\
\hline \multirow{2}{*}{ Calibration method in [11] } & Downlink & $3.731^{\circ}$ & $3.366^{\circ}$ & $3.211^{\circ}$ & $3.090^{\circ}$ & $2.209^{\circ}$ \\
\hline & Uplink & $4.397^{\circ}$ & $4.033^{\circ}$ & $3.370^{\circ}$ & $3.077^{\circ}$ & $2.900^{\circ}$ \\
\hline
\end{tabular}

According to [15], the normalized array antenna gain maintains to be larger than $98 \%$ as long as the phase error does not exceed $10^{\circ}$, which means that the power loss due to the mismatch of the antenna beam is not greater than $2 \%$ for the phase error being less than $10^{\circ}$. When the phase error is less than $3^{\circ}$, which is the case measured in our test-bed as mentioned above, the normalized array antenna gain is nearly 99.91\% [15], which means the proposed calibration method is nearly perfect for calibrating the array system like a massive MIMO which deals with the wide bandwidth LTE signals.

\section{Conclusion}

5G mobile communications have been rapidly evolving together with state-of-the-art technologies based on multiple antennas such as massive MIMO, Multiple User-MIMO, and so forth. In order to fully exploit the merits of the multiple antenna technologies, however, the transfer characteristics of each RF module should be accurately calibrated with one another. Furthermore, the calibration should be applicable to an extremely wide bandwidth signals like LTE or LTEAdvanced on real-time basis, which means the procedure should be valid while the target array system is transmitting or receiving the wide bandwidth data streams. This paper presented a novel method of equalizing the impulse responses of all the RF modules associated with a given antenna array system operating in an LTE signal environment. The proposed technique has demonstrates its superb performance for both downlink and uplink of LTE when it is applied to eNB array system. The proposed method uses the $\mathrm{Z}-\mathrm{C}$ sequence as a reference signal to estimate and compensate the impulse responses of the multiple RF modules in a given array system. From our experimental tests performed in our laboratory using an implemented test-bed, it has been found that the phase error between different RF modules does not exceed $3^{\circ}$. Recalling that the Z-C sequence is available in every data frame of LTE signals, it can be concluded that the proposed method is appropriate for calibrating the LTE-based array systems with a high accuracy in real-time.

\section{Competing Interests}

The authors declare that they have no competing interests.

\section{Acknowledgments}

This research was supported by the MSIP (Ministry of Science, ICT \& Future Planning), Korea, under the ITRC (Information Technology Research Center) support program (IITP-2015-H8501-15-1006) supervised by the IITP (Institute for Information \& communications Technology Promotion).

\section{References}

[1] T.-W. Ban and B. C. Jung, "A practical antenna selection technique in multiuser massive MIMO networks," IEICE Transactions on Communications, vol. 96, no. 11, pp. 2901-2905, 2013.

[2] W. Hong, K.-H. Baek, Y. Lee, Y. Kim, and S.-T. Ko, "Study and prototyping of practically large-scale mmWave antenna systems 
for $5 \mathrm{G}$ cellular devices," IEEE Communications Magazine, vol. 52, no. 9, pp. 63-69, 2014.

[3] F. Rusek, D. Persson, B. K. Lau et al., "Scaling up MIMO: opportunities and challenges with very large arrays," IEEE Signal Processing Magazine, vol. 30, no. 1, pp. 40-60, 2013.

[4] E. G. Larsson, O. Edfors, F. Tufvesson, and T. L. Marzetta, "Massive MIMO for next generation wireless systems," IEEE Communications Magazine, vol. 52, no. 2, pp. 186-195, 2014.

[5] S. Hyeon, Y. Yun, and S. Choi, "Novel automatic calibration technique for smart antenna systems," Digital Signal Processing, vol. 19, no. 1, pp. 14-21, 2009.

[6] H. Yamada, H. Sakai, and Y. Yamaguchi, "On array calibration technique for multipath reference waves," IEICE Transactions on Communications, vol. 94, no. 5, pp. 1201-1206, 2011.

[7] X. Hou, A. Harada, and H. Suda, "Experimental study of advanced MU-MIMO scheme with antenna calibration for the evolving LTE TDD system," in Proceedings of the IEEE 23rd International Symposium on Personal, Indoor and Mobile Radio Communications (PIMRC '12), pp. 2443-2448, IEEE, Sydney, Australia, September 2012.

[8] H. Fukuzono, T. Murakami, R. Kudo, S. Shinohara, Y. Takatori, and M. Mizoguchi, "Combining calibration schemes on a realtime multiuser MIMO-OFDM system with implicit feedback," in Proceedings of the 25th IEEE Annual International Symposium on Personal, Indoor, and Mobile Radio Communication (PIMRC '14), pp. 11-15, Washington, DC, USA, September 2014.

[9] S. Rangan, T. S. Rappaport, and E. Erkip, "Millimeter-wave cellular wireless networks: potentials and challenges," Proceedings of the IEEE, vol. 102, no. 3, pp. 366-385, 2014.

[10] L. L. Liou, D. M. Lin, M. Longbrake et al., "Digital wideband phased array calibration and beamforming using time reversal technique," in Proceedings of the 4th IEEE International Symposium on Phased Array Systems and Technology (ARRAY '10), pp. 261-266, Boston, Mass, USA, October 2010.

[11] M. Longbrake, L. L. Liou, D. M. Lin et al., "Wideband phased array calibration method for digital beamforming," in Proceedings of the IEEE National Aerospace and Electronics Conference (NAECON '12), pp. 11-17, IEEE, Dayton, Ohio, USA, July 2012.

[12] G. Tsoulos, J. McGeehan, and M. Beach, "Space division multiple access (SDMA) field trials. 2. Calibration and linearity issues," IEE Proceedings-Radar, Sonar and Navigation, vol. 145, no. 1, pp. 79-84, 1998.

[13] 3rd Generation Partnership Project, "Technical Specification Group Radio Access Network; Evolved Universal Terrestrial Radio Access (E-UTRA); Physical channels and modulation (Release 11)," 3GPP Organizational Partners TS 36.211 Rev. 11.3.0, 2013.

[14] R. Ziemer and W. H. Tranter, Principles of Communications: System Modulation and Noise, John Wiley \& Sons, New York, NY, USA, 2006.

[15] O. M. Bakr and M. Johnson, "Impact of phase and amplitude errors on array performance," Tech. Rep. UCB/EECS-2009-1, EECS Department, University of California, Berkeley, Berkeley, Calif, USA, 2009. 


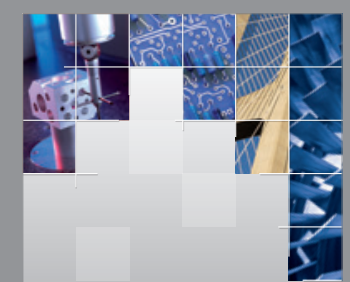

\section{Enfincering}
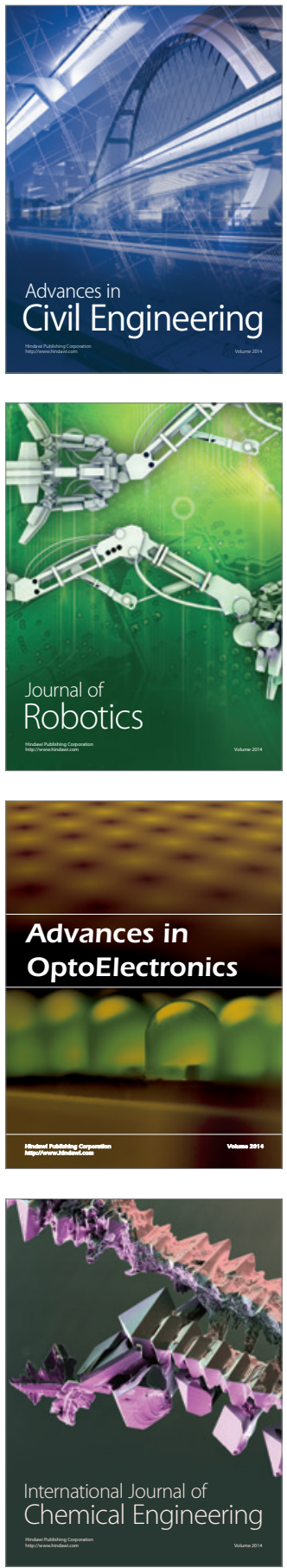

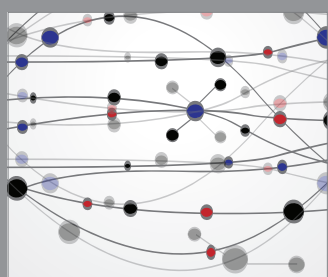

The Scientific World Journal

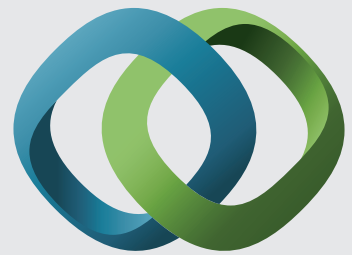

\section{Hindawi}

Submit your manuscripts at

http://www.hindawi.com
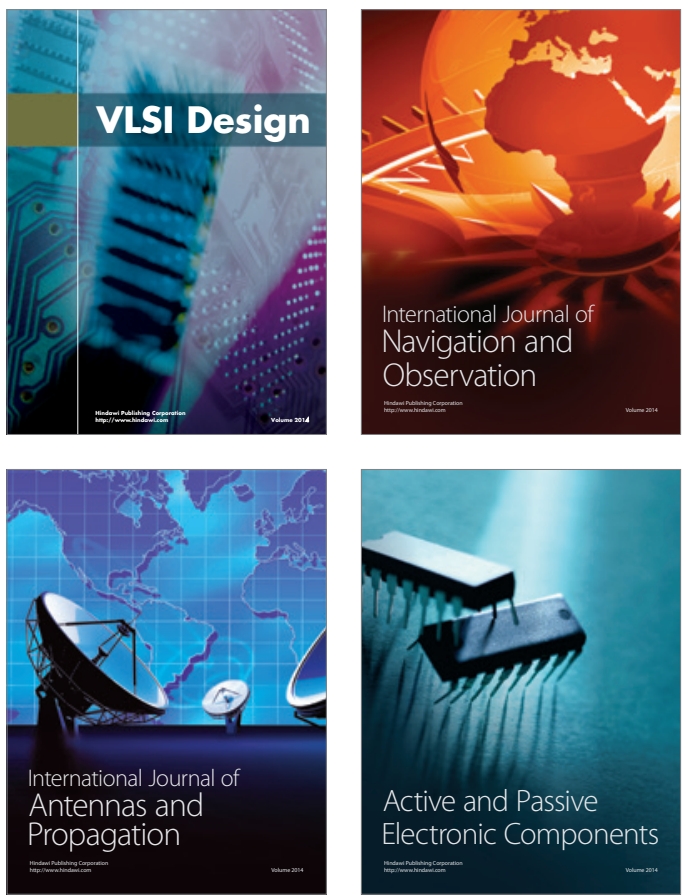
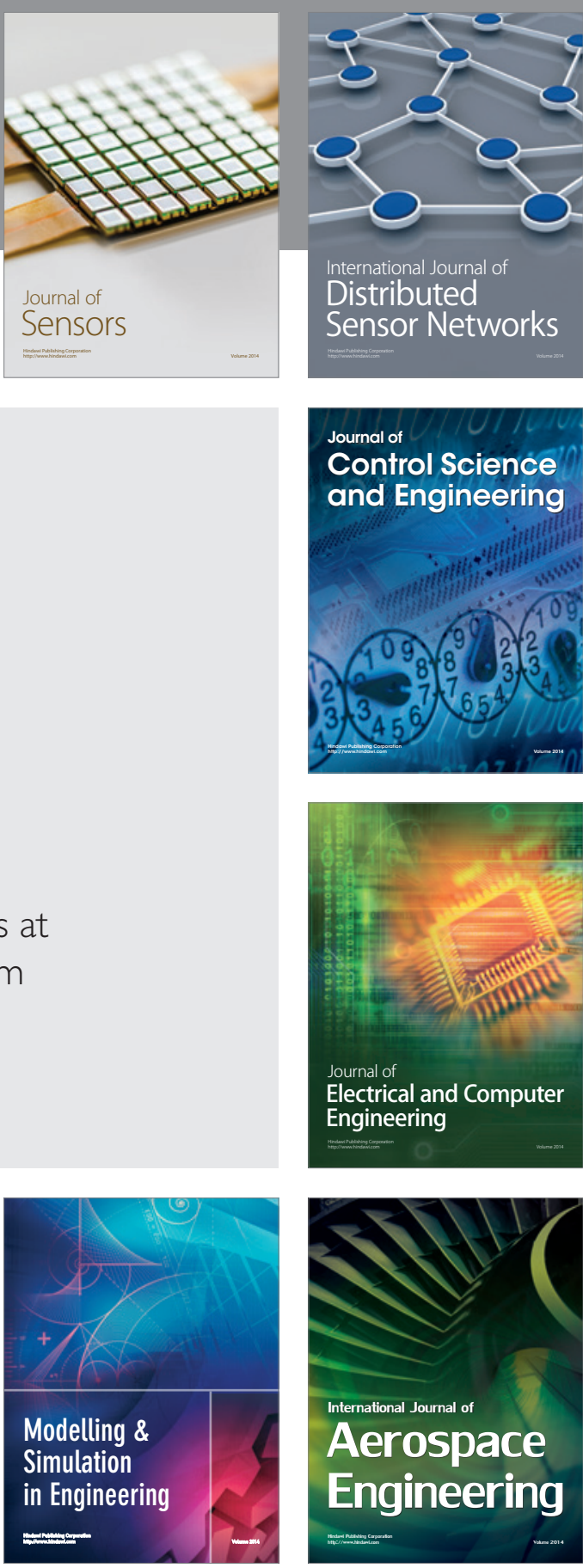

International Journal of

Distributed

Sensor Networks

Journal of

Control Science

and Engineering
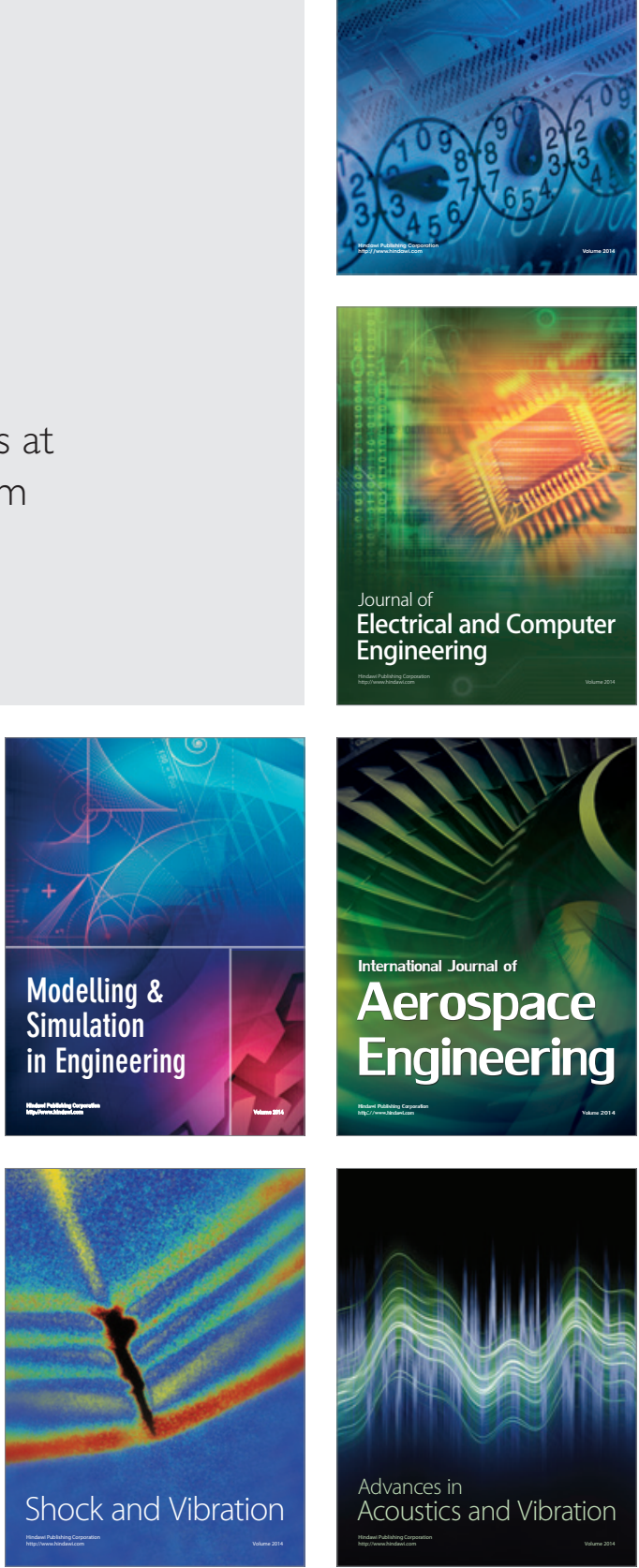